05

\title{
Магнитометрическая диагностика дефектов высокотемпературных сверхпроводящих лент в градиентном магнитном поле
}

\author{
() А.И. Подливаев, С.В. Покровский, И.В. Анищенко, И.А. Руднев \\ Национальный исследовательский ядерный университет МИФИ, \\ 115409 Москва, Россия \\ I e-mail: iarudnev@mephi.ru
}

(Поступило в Редакцию 18 мая 2018 г. В окончательной редакции 27 октября 2018 г.)

\begin{abstract}
Представлена новая методика бесконтактного магнитометрического определения локального критического тока в высокотемпературных сверхпроводящих лентах. В отличие от обычных подходов, где токи в сверхпроводнике наводятся однородным магнитным полем внешнего источника, в нашем варианте намагничивание ленты проведено линейкой разнополярных постоянных магнитов. Показано, что для ряда технических задач диагностики дефектов предложенный нами подход эффективнее применявшихся ранее. Рассмотрены два варианта диагностики. Первый предназначен для экспрессной диагностики локальных дефектов (в первую очередь поперечных трещин) в отдельной ленте и в стопке лент, второй разработан для случая гладкого изменения критического тока отдельной ленты. Предлагаемый метод позволяет существенно повысить точность определения плотности локального критического тока.
\end{abstract}

DOI: 10.21883/JTF.2019.04.47307.197-18

\section{Введение}

Для применения высокотемпературных сверхпроводников (ВТСП) в кабелях, токоограничителях, моторах, генераторах и т.п. необходимы достаточно длинные гибкие токонесущие элементы - ВТСП-ленты второго поколения (2G). 2G ВТСП-ленты представляют собой тонкий (толщина $d$ порядка $1 \mu \mathrm{m}$ ) слой ВТСП, нанесенный с использованием набора промежуточных буферных слоев на гибкую металлическую подложку [1]. Одной из основных целей совершенствования $2 \mathrm{G}$ ВТСП-лент является достижение высоких однородных значений критического тока $I_{c}$ по всей длине ленты, которая может достигать нескольких сотен метров, в отдельных случаях - до километра. Эта задача требует применения различных методов контроля однородности $I_{c}$. Прямые измерения транспортного критического тока четырехконтактным методом на последовательных локальных участках ленты затруднительны и трудоемки. Кроме того, транспортные измерения дают лишь усредненное значение критического тока областей, локализованных между потенциальными контактами, и не отражают двумерное пространственное распределение плотности критического тока $j_{c}$. Для экспресс-диагностики однородности критического тока могут быть использованы бесконтактные методы. К таким методам можно отнести измерение величины магнитного поля, захваченного сверхпроводником, а также левитационную методику, основанную на измерении силы взаимодействия постоянного магнита с токами, индуцированными в сверхпроводнике.

Для детальной диагностики неоднородностей токонесущих характеристик сверхпроводящих лент применяются прецизионные магнитометрические методики, основанные на бесконтактном измерении пространствен- ного распределения магнитного поля на мелкой пространственной сетке над всей поверхностью ВТСПленты. Для оценки величины плотности критического тока в ВТСП-ленте внешним магнитным полем (ВМП) наводятся токи. По магнитному полю наведенных токов посредством различных алгоритмов (см. обзор [2] и ссылки в нем) восстанавливается плотность токов в ленте, что в случае достаточно большого значения ВМП (используя модель критического состояния $[3,4]$ ) позволяет определить наличие сверхпроводящих неоднородностей ленты и оценить локальный транспортный ток. При простейшей диагностике для наведения токов в изучаемой ленте чаще всего применяется изменяющееся во времени, но пространственно однородное магнитное поле или поле одиночного магнита, над которым лента протягивается в процессе контроля ее качества. При этом достаточно измерять распределение магнитного поля над лентой путем сканирования измерительным элементом (например, преобразователем Холла) с шагом, малым по сравнению с шириной ленты. Этот простейший подход имеет ряд недостатков, которые будут обсуждены ниже и которые можно обойти, если в качестве индуктора токов в ВТСП-ленте использовать применяемую в настоящей работе линейку магнитов различной поляризации. Линейка магнитов является общим элементом двух различных взаимодополняющих методик диагностики дефектов различного типа.

Первая методика подразумевает численное нахождение сверхпроводящих токов из уравнения Био-Савара и из материальных уравнений сверхпроводника, что необходимо для определения магнитного поля и плотности сверхпроводящих токов, а также вычисление силы левитации между сверхпроводником и линейкой разнополяризованных магнитов. При этом рассчитывается сила взаимодействия как для бездефектного ВТСП- 
образца, так и для образца с дефектами. Сопоставление экспериментально измеренной силы левитации с расчетными данными позволяет сделать вывод о типе и местоположении дефекта.

Во втором подходе (методике) рассматривается обратная задача Био-Савара, при решении которой определяется распределение плотности сверхпроводящих токов в ВТСП-ленте. Исходной информацией для этой задачи является экспериментально измеренное (холловская магнитометрия) магнитное поле остаточной намагниченности над всей поверхностью сверхпроводящей ленты. В качестве индуктора остаточной намагниченности в предлагаемом нами варианте методики также применяется линейка магнитов.

Представленная совокупность методик позволяет проводить магнитометрическую и левитационную диагностики локализованных дефектов (трещины, несверхпроводящие включения и т.п.) в отдельных лентах и в стопках лент, а также диагностику слабых неоднородностей сверхпроводящих параметров ВТСП-лент.

\section{1. Основные уравнения}

\section{1. Связь магнитного, электрического поля и наведенного тока в сверхпроводнике}

Базовым элементом магнитометрических методик является закон Био-Савара, связывающий вектор полной магнитной индукции $\mathbf{B}(\mathbf{r})$ в точке пространства с радиус-вектором $r$, вектор магнитной индукции внешних источников поля $\mathbf{B}_{\text {ext }}(\mathbf{r})$, и вектор $\mathbf{j}(\mathbf{r})$ - плотность сверхпроводящего (СП) тока в ВТСП-ленте:

$$
B(r)=B_{\text {ext }}(r)+\frac{\mu_{0}}{4 \pi} \int_{V} \frac{\mathbf{j}\left(r^{\prime}\right) \times\left(\mathbf{r}-\mathbf{r}^{\prime}\right) d^{3} \mathbf{r}^{\prime}}{\left|\mathbf{r}-\mathbf{r}^{\prime}\right|^{3}},
$$

где $\mu_{0}$ - магнитная проницаемость вакуума, а интегрирование ведется по всему объему сверхпроводящей части ленты. Полагаем, что в пространственных координатах $(X, Y, Z)$ поверхность ВТСП-ленты (а также стопки лент) расположена перпендикулярно оси $Z$, причем центр ленты (стопки лент) имеет нормальную координату $Z=0$.

В отсутствие транспортного тока в ленте линии сверхпроводящих токов, индуцированных внешним магнитным полем, замкнуты. Полагаем, что скорость изменения внешнего магнитного поля достаточно мала, чтобы токи, наведенные в несверхпроводящей подложке ленты, также были пренебрежимо малы. Это позволяет трансформировать уравнение (1) в более удобное уравнение для квазистационарной задачи, связывающее нормальные компоненты индукции $B_{Z}(\mathbf{r}, t), B_{Z \operatorname{ext}}(\mathbf{r}, t)$ с нормальной компонентой вектора намагниченности ВТСПленты $\mathbf{g}(X, Y)=\left(g_{X}, g_{Y}, g_{Z}\right)$ (см. [2,5]). Плотность тока при этом определяется выражением (2):

$$
\mathbf{j}(X, Y, t)=\nabla \times \mathbf{g}(X, Y, t) .
$$

Для сверхпроводящей пленки компоненты вектора намагниченности в плоскости $(X, Y)$ равны нулю $\left(g_{X}=g_{Y}=0\right)$, в то время как компонента $g_{Z}$ в направлении оси $Z$ равна $g_{Z}=g(X, Y, t)([2,5])$. В рамках сделанных предположений из уравнений (1) и (2) мы получаем связь магнитного момента и нормальных компонент полного и внешнего магнитных полей в момент времени $t$ :

$$
\begin{gathered}
B_{Z}(X, Y, Z, t)=B_{\mathrm{ext}}(t y)+\mu_{0} \int_{S} K\left(r, r^{\prime}\right) g\left(X^{\prime}, Y^{\prime}, t\right) d X^{\prime} d Y^{\prime}, \\
K\left(r, r^{\prime}\right)=\frac{1}{4 \pi} \frac{\left.2 Z-\left(X-X^{\prime}\right)\right)^{2}-\left(Y-Y^{\prime}\right)^{2}}{\left[Z^{2}+\left(X-X^{\prime}\right)^{2}+\left(Y-Y^{\prime}\right)^{2}\right]^{5 / 2}}
\end{gathered}
$$

где $K\left(r, r^{\prime}\right)$ - ядро интегрального оператора.

Связь магнитного $\mathbf{B}(X, Y, Z, t)$ и электрического $\mathbf{E}(X, Y, Z, t)$ полей определяется законом индукции

$$
\frac{\partial \mathbf{B}(X, Y, Z, t)}{\partial t}=-\nabla \times \mathbf{E}(X, Y, Z, t) .
$$

Для сверхпроводящей пленки, перпендикулярной оси $Z$, выражение (4) дает

$$
\frac{\partial B_{Z}(X, Y, Z, t)}{\partial t}=-\frac{\partial E_{X}(X, Y, Z, t)}{\partial Y}+\frac{\partial E_{Y}(X, Y, Z, t)}{\partial X} .
$$

Для решения прямой задачи нам необходимо задать материальные уравнения - связь электрического поля и тока в сверхпроводнике. Несверхпроводящую часть ВТСП-ленты мы считаем диэлектриком вследствие медленного квазистационарного изменения внешнего магнитного поля. Определим нелинейный закон Ома для сверхпроводящей пленки следующим образом:

$$
\mathbf{E}(X, Y, 0, t)=\rho(\mathbf{j}(X, Y, t), B(X, Y, 0, t)) \cdot \mathbf{j}(X, Y, t) .
$$

Зависимость удельного сопротивления $\rho(\mathbf{j}, B)$ часто выбирают в следующем виде:

$$
\rho(\mathbf{j}, B)=\rho\left[|\mathbf{j}| / j_{c}(B)\right]^{n},
$$

где $n \sim 20$, а зависимость плотности критического тока от амплитуды магнитного поля $j_{c}(B)$ определяется в одной из моделей критического состояния (см. обзор [2] и ссылки в нем). Выражение (7) является часто используемым, но, как указано в работе [5], не единственным. Для применения приближения (7) требуется задание трех параметров: $\rho_{0}, n$ и скорости изменения внешнего магнитного поля. При определении этих параметров получающаяся математическая задача позволяет описать временну́ю зависимость поля наведенных токов при различной скорости изменения внешнего магнитного поля, а также исследовать процессы релаксации токов после того, как внешнее магнитное поле после изменения было зафиксировано. Нас, однако, интересует случай адиабатически медленного изменения внешнего магнитного 
поля (предел скорости изменения внешнего поля, равный нулю). Этот случай наиболее точно соответствует условиям реальных экспериментов. Для описания такого предельного случая приближение (7) непригодно, поскольку обладает конечным сопротивление любой точки ленты в токовом состоянии. В этой ситуации нулевая скорость изменения внешнего поля приведет к нулевому значению плотности наведенных токов. Для выхода из этой формальной проблемы в настоящей работе, также как в работах $[6,7]$, мы выбираем зависимость $\rho(j, B)$ следующим образом:

$$
\rho(\mathbf{j}, B)=\left\{\begin{array}{ll}
0, & \text { for }|\mathbf{j}|<j_{c}(B) \\
\rho_{0}\left[|\mathbf{j}|-j_{c}(B)\right]^{2}, & \text { for } j_{c}(B) \leq|\mathbf{j}| \leq 2 j_{c}(B)
\end{array} .\right.
$$

Обоснование вида зависимости (8) заключается в следующем. При выборе этой зависимости и адиабатически медленном нарастании внешнего магнитного поля параметр $\rho_{0}$ не влияет на решение задачи, поскольку в уравнениях (5), (6) время может быть перенормировано на $\rho_{0}$. Также в этом пределе на решение не влияет квадратичный показатель степени над разностью в выражении $(8) \rho_{0}\left[|\mathbf{j}|-j_{c}(B)\right]^{2}$. При адиабатически медленном изменении внешнего поля в соответствии с моделью критического состояния $[3,4]$ плотность СП-тока ленты $j$ может достигать (но не превышать) критического значения $j_{c}(B)$. Показатель степени в зависимости (8) хотя и не влияет на конечный результат расчетов, но сильно влияет на устойчивость и скорость сходимости итерационного алгоритма численного решения данной задачи. Вид выражения (8) выбирался так, чтобы численное решение задачи требовало минимальных затрат компьютерного времени. Апробация данного подхода, проведенная в работах [6,7], показала его эффективность.

Для постановки прямой задачи необходимо также учитывать полевую зависимость плотности критического тока от величины магнитного поля в сверхпроводнике. К настоящему времени разработано множество моделей критического состояния с различными функциональными зависимостями критического тока от магнитного поля - от простейшей модели Бина, где эта зависимость вообще отсутствует, до моделей с многопараметрическими зависимостями, которые анализируются в работе [8]. В наших дальнейших расчетах мы будем использовать предложенную в [8] двухэкспоненциальную модель (double exponential model DEM), которая позволяет с высокой точностью описать экспериментальную зависимость $j_{c}(B)$ в диапазоне магнитного поля от нуля до $6 \mathrm{~T}$. В этой модели локальная плотность критического тока определяется следующим выражением:

$$
j_{c}(B)=A_{1} \exp \left(-|B| \beta_{1}\right)+A_{2} \exp \left(-|B| \beta_{2}\right) .
$$

В настоящей работе мы используем следующие значения параметров, экспериментально определенные в [8]: $A_{1}=12.9 \mathrm{kA} / \mathrm{m}, A_{2}=13.8 \mathrm{kA} / \mathrm{m}, \beta_{1}=0.08 \mathrm{~T}, \beta_{2}=1.92 \mathrm{~T}$.
В результате мы получаем систему уравнений $(2)-(6),(8),(9)$ для определения величины $g(X, Y, t)$, токов $j(X, Y, t)$ и нормальной компоненты магнитного поля $\mathbf{B}(X, Y, 0, t)$ на поверхности сверхпроводящей пленки. Эти уравнения для прямой задачи дополняются нулевыми начальными условиями $g(X, Y, t=0)=0$, и условием равенства нулю магнитного момента вне ленты $g(X, Y, t)=0$, что завершает математическую постановку прямой задачи.

Для эффективного численного решения как прямой, так и обратной задачи необходимо фурьепреобразование уравнения (3). В результате преобразования для фурье-образов получаем уравнение (10):

$$
\begin{aligned}
\tilde{B}\left(k_{X}, k_{Y}, Z, t\right)= & \tilde{B}_{\text {ext }}\left(k_{X}, k_{Y}, Z, t\right) \\
& +\mu_{0} \tilde{K}\left(k_{X}, k_{Y}, Z\right) \tilde{g}\left(k_{X}, k_{Y}, t\right) .
\end{aligned}
$$

Соответствующие компоненты магнитного поля и момента связаны с их фурье-образами следующими соотношениями:

$$
\begin{aligned}
& \tilde{B}_{Z}\left(k_{X}, k_{Y}, Z, t\right)=\int_{-\infty}^{+\infty} d X \int_{-\infty}^{+\infty} d Y \\
& \times \exp \left(i k_{X} X+i k_{Y} Y\right) B_{Z}(X, Y, Z, t), \\
& \tilde{g}\left(k_{X}, k_{Y}, t\right)=\int_{-\infty}^{+\infty} d X \int_{-\infty}^{+\infty} d Y \\
& \times \exp \left(i k_{X} X+i k_{Y} Y\right) g(X, Y, t) .
\end{aligned}
$$

Для малых толщин сверхпроводящего слоя $d$ фурьеобраз ядра интегрального оператора $\tilde{K}\left(k_{X}, k_{Y}, Z\right)$ определяется следующим выражением [9]:

$$
\tilde{K}\left(k_{X}, k_{Y}, Z\right)=\exp (-k|Z|) k / 2, \quad k=\sqrt{f_{X}^{2}+k_{y}^{2}} .
$$

Прямая задача (3)-(6), (8)-(12) решается численно, детали численных алгоритмов решения даны в рабо$\operatorname{Tax}[6,7]$.

\section{2. Индукция внешнего поля линейки разнополяризованных магнитов}

При решении прямой задачи (3)-(11) об определении намагниченности ВТСП-ленты и силы взаимодействия ленты и линейки магнитов значение внешнего поля $\mathbf{B}_{\text {ext }}(\mathbf{r}, t)$ отлично от нуля и изменяется с течением времени. При решении обратной задачи уравнения (1) внешнее поле $\mathbf{B}_{\text {ext }}(\mathbf{r}, t)=0$. В реальном эксперименте c использованием линейки разнополярных постоянных магнитов равенство нулю внешнего поля обеспечивается отводом линейки на достаточно большое расстояние, при котором внешнее поле постоянных магнитов на уровне ВТСП-ленты становится несущественным. Однако очевидно, что остаточная намагниченность ВТСПленты, индуцированная линейкой магнитов, отражает 
особенности магнитного поля этой линейки. Рассмотрим распределение магнитного поля от линейки магнитов.

В линейке магнитов, как уже отмечалось, соседние магниты имеют противоположную полярность, и вектор создаваемого ими магнитного поля на поверхности магнита направлен вдоль оси $Z$. Полагаем, что продольный размер каждого магнита существенно превышает ширину ленты, и (в направлении оси $X$ ) в рамках нашей модели считается неограниченным. Длина магнитной линейки в направлении оси $Y$ также считается неограниченной. Аналогичная геометрия линейки предлагалась ранее при кратком описании диагностики слабых неоднородностей ВТСП-ленты [10].

В модели взаимодействия ВТСП-ленты с магнитами полагаем, что нормальная компонента магнитного поля, создаваемого линейкой, является величиной постоянной и равной $B_{Z}= \pm B_{\max }$ над каждым магнитом и равной нулю на их стыках, значение этой компоненты на поверхности линейки может быть представлено в следующем виде:

$$
\mathbf{B}_{Z \operatorname{ext}}(X, Y, Z=0)= \begin{cases}B_{\max }, & \text { for } \sin (\pi Y / a)>0, \\ 0, & \text { for } \sin (\pi Y / a)=0, \\ -B_{\max }, & \text { for } \sin (\pi Y / a)<0 .\end{cases}
$$

В этом случае в полупространстве $Z>0$ периодическая вдоль оси $Y$ и убывающая по переменной $Z$ гармоническая функция $B_{Z}(X, Y, Z)$ имеет следующий вид:

$$
\begin{gathered}
\mathbf{B}_{Z \text { ext }}(X, Y, Z)=\sum_{n=0,1,2 \ldots} B_{n}(-1)^{n} \sin \left[\varphi_{n} Y\right] \exp \left[-\varphi_{n} Z\right], \\
B_{n}=4 B_{\text {max }} /[\pi(2 n+1)], \varphi_{n}=\pi(2 n+1) / a .
\end{gathered}
$$

В линейке реальных магнитов разрыв поля на стыке сглажен. Также следует учитывать, что в реальных левитационных экспериментах сила взаимодействия измеряется не в режиме касания постоянных магнитов и ВТСП-ленты, а на некотором удалении магнитов от ленты. Поэтому для описания магнитного поля линейки мы в дальнейшем ограничимся основным (нулевым) слагаемым суммы (14):

$$
\mathbf{B}_{Z \operatorname{ext}}(X, Y, Z)=B_{0} \sin \left[\varphi_{0} Y\right] \exp \left[-\varphi_{0} Z\right] .
$$

Поскольку в линейке предполагается использовать постоянные магниты $\mathrm{NdFeB}$, считаем в дальнейшем величину $B_{0}=0.3 \mathrm{~T}$, которая является характерной для магнитов такого типа.

Достаточно простой вид магнитного поля (15) позволяет определить нормальную компоненту левитационной силы $F_{Z}$ взаимодействия ВТСП-ленты с линейкой следующим образом:

$$
\begin{aligned}
F_{Z} & =\int_{S} g(X, Y) \frac{\partial \mathbf{B}_{Z \operatorname{ext}}(X, Y, Z)}{\partial Z} d X d Y \frac{\partial \mathbf{B}_{Z \text { ext }}(X, Y, Z)}{\partial Z} \\
& =-\varphi_{0} B_{0} \sin \left[\varphi_{0} Y\right] \exp \left[-\varphi_{0} Z\right]=-\varphi_{0} \mathbf{B}_{Z \operatorname{ext}}(X, Y, Z) .
\end{aligned}
$$

Интегрирование проводится по поверхности ВТСПленты.

\section{2. Диагностика дефектов в стопках ВТСП-лент с помощью левитационной методики}

Одним из направлений технического применения высокотемпературных сверхпроводников является создание различных устройств, основанных на явлении магнитной левитации. К настоящему времени продемонстрированы возможности создания левитационного транспорта на открытом воздухе и в вакуумной трубе $[11,12]$, созданы действующие полномасштабные прототипы левитационных подшипников [13]. В этих случаях подъемная сила (сила левитации) подвеса обусловлена взаимодействием внешнего магнита с токами, индуцированными магнитным полем в сверхпроводнике. Внешнее поле при этом должно быть неоднородным в направлении создаваемой подъемной силы, для чего часто используется конфигурация постоянных магнитов, называемая массивом Халбаха (Halbach). Примеры использования массива Халбаха можно найти в [11-13]. До настоящего времени в качестве сверхпроводящих элементов, как правило, применялись объемные ВТСПматериалы, недостатками которых является низкая механическая прочность, относительно невысокая плотность критического тока, постепенная потеря сверхпроводящих свойств при воздействии паров воды, а главное, значительная трудность оптимизации масс-габаритных параметров устройств. Последнее обстоятельство связано с тем, что объемные высокотемпературные сверхпроводники чрезвычайно трудно поддаются механической обработке без деградации характеристик. Между тем все эти недостатки отсутствуют у современных ВТСПлент второго поколения, что делает их перспективными кандидатами для использования в левитационных устройствах при условии замены объемных ВТСП стопками ВТСП-лент. В настоящее время активные исследования левитационных характеристик массивов из ВТСП-лент позволили выявить основные свойства таких систем, а именно зависимости левитационной силы при вертикальных и латеральных смещениях, влияние знакопеременных механических воздействий, влияние скрещенного магнитного поля и т.п. [14-18].

Следует отметить, что на практике создание градиентного поля с помощью массива Халбаха достаточно трудоемко и затратно, учитывая необходимость использования большого числа постоянных магнитов на основе редкоземельных металлов. В разд. 2 рассмотрен упрощенный подход, основанный на взаимодействии стопки сверхпроводящих лент с плоской линейкой разнополярных магнитов, заменяющих более сложную конфигурацию массива Халбаха. Для этой конфигурации нами проведен расчет распределения токов в ленте, наведенных линейкой магнитов (решение задачи (3)-(6), (8)-(15)), 


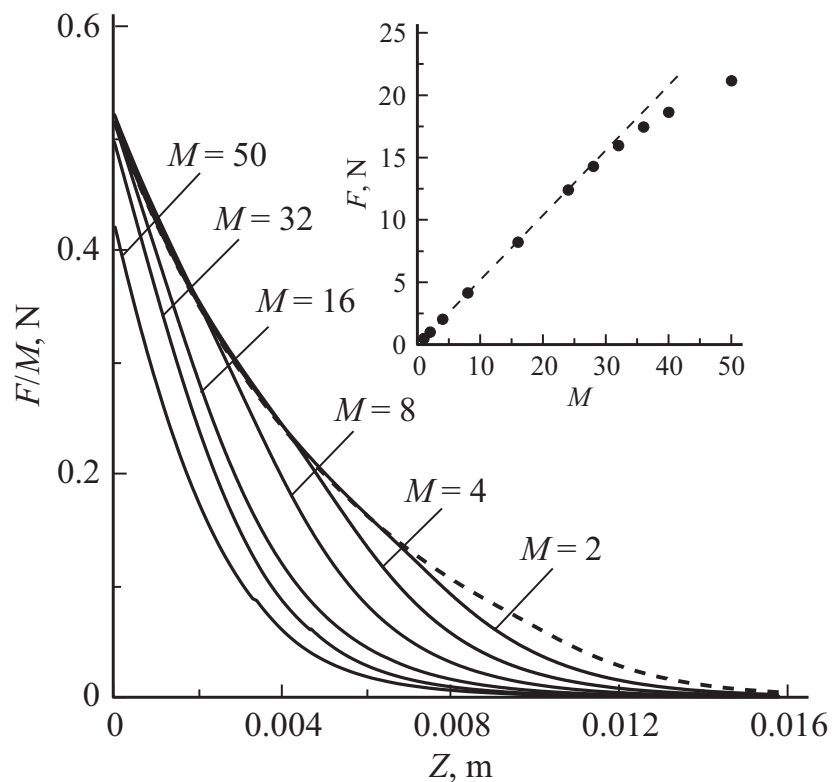

Рис. 1. Зависимость силы левитации $F / M$, приходящуюся на одну ленту стопки для фрагмента стопки размером $12 \times 24 \mathrm{~mm}$. $M$ - количество лент в стопке. Штриховой линией на основном рисунке обозначена зависимость силы левитации уединенной ленты. На вставке представлена зависимости силы левитации от числа лент в стопке при $Z=0$.

определена сила левитации для стопки, состоящей из различного числа ВТСП-лент на основе выражения (16). Также впервые определено влияние на силу взаимодействия наличия технологического дефекта - поперечной трещины в сверхпроводящем слое одной из лент в стопке, что указывает на возможность использования левитационных методик для диагностики дефектов в ВТСП-лентах.

Полагаем ширину ленты в направлении оси $X$, как и ширину каждого отдельного магнита в направлении оси $Y$, равной величине $a=12 \mathrm{~mm}$. Кроме силы взаимодействия линейки магнитов с отдельной ВТСПлентой определялось взаимодействие со стопкой лент при определении полного магнитного момента стопки квадратных фрагментов ленты. Количество лент в стопке $M$ при наших расчетах было равно 1, 2, 4, 8, 16, 24, $28,32,36,40$ и 50.

Проведенные нами расчеты показали, что сила взаимодействия отдельной ленты в стопке с магнитной линейкой практически не зависит от количества лент в стопке, если это количество не превышает 30. Этот результат коррелирует с данными экспериментальной работы [14], где полная сила левитации стопки лент в неоднородном магнитном поле была пропорциональна количеству ВТСП-лент вплоть до 10. При сопоставлении данных необходимо учитывать, что в экспериментальной работе [14] расстояние до магнита отсчитывалось от поверхности стопки, а в настоящей работе - от ее (стопки) середины. Действительно, на рис. 1 представ- лена зависимость силы взаимодействия с магнитной линейкой отдельной ленты и стопок из 2, 4, 8, 16, 32 и 50 лент от нормальной координаты $Z$ и количества лент в стопке $M$. На рисунке видно, что при $Z=0$ сила левитации, приходящаяся на одну ленту стопки, не изменяется вплоть до количества лент в стопке $M \sim 30$, однако при большем удалении стопки от магнитной линейки зависимость от количества лент заметна, что связано с экранировкой внешнего поля сверхпроводящими лентами.

Одним из часто встречающихся дефектов ВТСПленты, возникающим в процессе изготовления, является поперечная трещина сверхпроводящего слоя. Мы рассчитали уменьшение левитационной силы из-за наличия трещины в одной из лент стопки и нашли, что в случае, когда поперечная трещина расположена над границей раздела двух магнитов линейки, сила левитации не меняется. Этот результат обусловлен тем, что, поскольку трещина параллельна токовым линиям, она не влияет на процесс намагничивания стопки (рис. 2, $a$, где границы раздела магнитов имеют координаты $Y=0, \pm 12 \mathrm{~mm}$ и т. д.).

Однако если трещина расположена над серединой магнита, ситуация кардинально изменяется. На рис. 2, $b$, с представлены линии тока в уединенной ленте с трещиной и в пакете из двух лент, одна из которых имеет трещину. Изменение токовой структуры приводит к уменьшению силы магнитной левитации. На рис. 3 представлена разность сил левитации $F(Z, M)-F_{d e f}(Z, M)$ бездефектной стопки лент $(F(Z, M))$ и стопки с одной дефектной лентой $\left(F_{d e f}(Z, M)\right)$ в зависимости от расстояния $Z$ при различном количестве $M$ лент в стопке. На этом рисунке видно, что с ростом числа лент в стопке влияние дефектной ленты ослабевает и становится более короткодействующим. Так, при увеличении числа лент в стопке от одной до 32 изменение силы левитации за счет дефектной ленты падает в 3 раза при $Z=0$, а при $Z=4 \mathrm{~mm}$ не регистрируется вовсе.

Изменение силы левитации из-за поперечной трещины одной ленты в стопке может быть использовано для определении факта наличия этой трещины, а зависимость силы от взаимного расположения трещины и границы разнополярных магнитов линейки позволяет определить конкретное местоположение трещины в стопке. Сопоставление рис. $2, a, b$ показывает, что трещина изменяет не только область над магнитом, над которым находится, но и структуру токов в соседних областях. Однако вблизи трещины это изменение носит радикальный характер, в то время как в соседних областях изменение является небольшой поправкой. Это обстоятельство позволяет применять данный подход не только для диагностики трещин в протяженных ВТСПлентах. Также возможна адаптация методики к диагностике трещин в изделиях, состоящих из прямоугольных участков СП пленки, разделенных несверхпроводящими полосами (например, к микроволновому делителю для квантовых сверхпроводящих цепей [19]). 

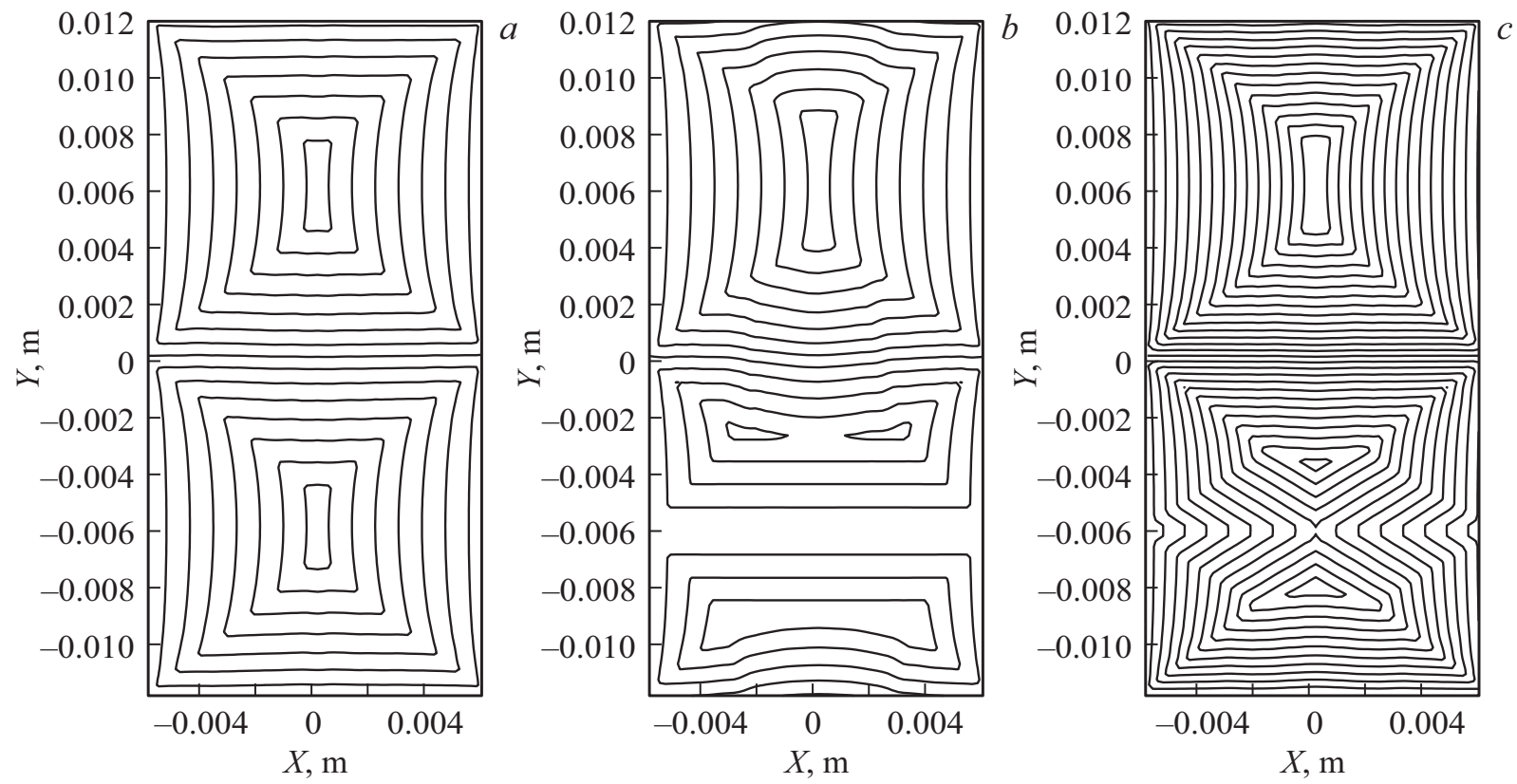

Рис. 2. Линии наведенных токов в фрагменте ВТСП-ленты. Каждая линия соответствует току, равному 10 А: $a-$ в отдельной ленте без дефектов; $b-$ в отдельной ленте с поперечной трещиной (координата $Y=-0.006 \mathrm{~m}$ ); $c$ - суммарный ток в стопке из двух лент, одна из которых имеет трещину (координата $Y=-0.006 \mathrm{~m}$ ).

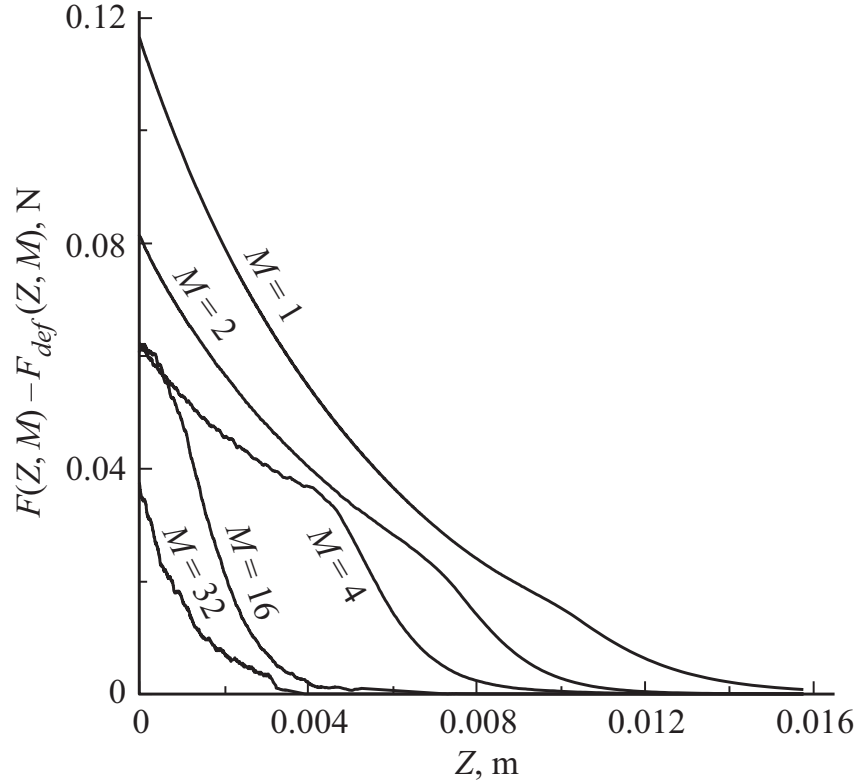

Рис. 3. Зависимость изменения силы левитации, обусловленное поврежденной лентой в стопке, от расстояния $Z$ при различном количестве $M$ лент в стопке.

\section{3. Прецизионная магнитометрическая диагностика гладких неоднородностей критического тока в ВТСП-лентах}

Задачи диагностики ВТСП-ленты можно условно разбить на различные классы в зависимости от ожидае- мого характера дефектов ленты. В разд. 3 в отличие от предыдущего нас интересует магнитометрическое определение локального критического тока в случае, когда значение критического тока изменяется гладко, без разрывов, а размер неоднородностей сопоставим с шириной ленты. Такого рода дефекты могут появляться в результате несовершенств технологического процесса, возникающих, например, при медленном отклонении параметров нанесения ВТСП-слоя от оптимальных значений. Решение этих задач представляет интерес как метод контроля качества производимых ВТСП-лент.

В данном разделе представлено восстановление по магнитному полю $\mathbf{B}(\mathbf{r})$ токов $\mathbf{j}$ остаточной намагниченности. Единственным источником магнитного поля после удаления поля внешнего источника остаются наведенные токи, связь которых с нормальной компонентой (ось $Z$ ) дается законом Био-Савара (1) при $\mathbf{B}_{\mathrm{ext}}(\mathbf{r})=0$.

Обратная задача (1) определения плотности тока по магнитному полю относится к классу некорректных задач, решение которых требует специальных подходов [20]. Проблема восстановления тока $\mathbf{j}(\mathbf{r})$ по магнитному полю $\mathbf{B}(\mathbf{r})$ обусловлена тем, что из-за интегрирования в (1) пространственно неоднородные коротковолновые гармоники (КВГ) тока $\mathbf{j}(\mathbf{r})$ слабо влияют на магнитное поле, вследствие чего не могут быть точно из него определены. Существуют разные методики решения данной задачи, но их общим и обязательным элементом является регуляризация (искусственное сглаживание) определяемых токов $[2,16]$. Регуляризация заключается в применении фильтрующих функций, которые подавляют КВГ-шумы, приводящие к существенным ошибкам при 


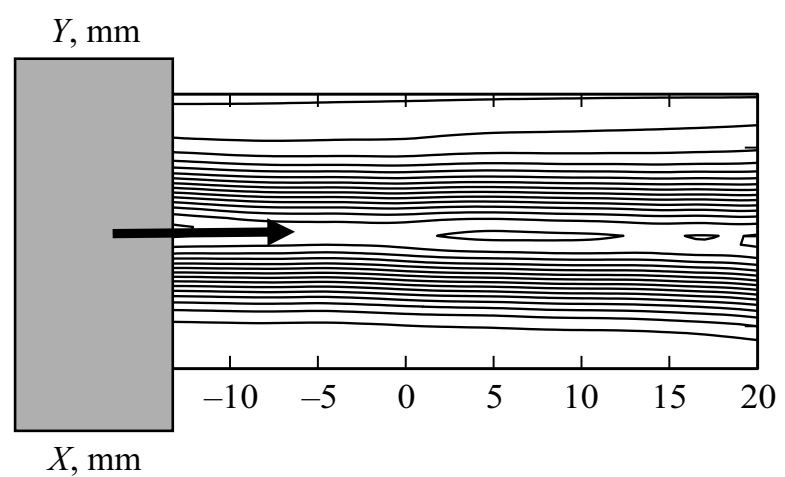

Рис. 4. Схема продольного намагничивания ВТСП-ленты и наведенные токовые линии в ней. Серым прямоугольником обозначен постоянный магнит. Стрелка указывает направление движения магнита при намагничивании. Приведен участок с продольным размером $40 \mathrm{~mm}$ в длинной намагниченной ленте. Каждая токовая линия соответствует току $3 \mathrm{~A}$.

решении. Вследствие регуляризации для пары исходного (не сглаженного) экспериментального В $(\mathbf{r})$ и восстановленной (сглаженной) плотности тока $\mathbf{j}(\mathbf{r})$ закон БиоСавара выполняется приближенно, однако полученные таком образом результаты вполне адекватно описывают реальное распределение токов.

Так же как в разд. 2, наиболее удобным методом решения обратной задачи относительно магнитного момента является применение преобразования Фурье (10) к обеим частям уравнения (3). В результате фурьеобраз магнитного момента ленты выражается через фурье-образ магнитного поля посредством уравнения (10). Намагничивание ВТСП-ленты часто проводится пространственно-однородным магнитным полем [2] или его аналогом - намагничиванием при продольном пе-

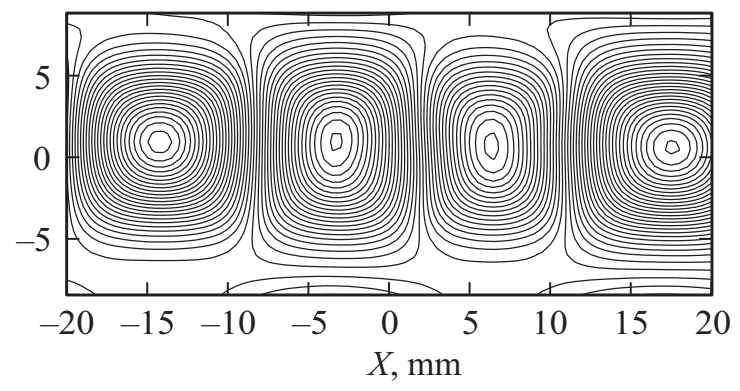

ремещением ленты над постоянным магнитом. Относительное расположение образца и магнита в стандартной схеме представлено на рис. 4.

На этом рисунке представлены также токовые линии, полученные из магнитного поля остаточной намагниченности ВТСП фрагмента ленты фирмы СуперОкс с шириной $L_{Y}=12 \mathrm{~mm}$. Методика намагничивания постоянным магнитом (с индукцией $0.3 \mathrm{~T})$ и восстановления токов стандартная. Данный подход содержит ряд недостатков, два из которых явно видны из формы токовых линий рис. 4.

Первый недостаток заключается в том, что при данной методике наведенный ток вблизи центральной продольной линии ниже критического, а в центре ленты обращается в нуль (подробнее см. обзор [2] и литературу к нему). Причина связана с однородным характером намагничивания образца. Вследствие такого типа намагничивания данная методика не позволяет определить величину плотности критического тока в центральной части ленты. Одновременное воздействие магнитного поля и транспортного тока может снять эту проблему, поскольку приложение транспортного тока смещает область малых токов от центра к периферии [7]. Однако одновременное намагничивание и пропускание транспортного тока через ленты технически затруднительно, особенно для длинных лент.

Второй недостаток заключается в низкой точности определения плотности наведенного тока вблизи границы ленты. На рис. 4 видно, что плотность тока сравнительно гладко спадает вблизи границы ленты и отлична от нуля даже за пределами образца (что является артефактом). Причина возникновения эффекта „размытой“ границы обусловлена регуляризацией - подавлением КВГ, без которых невозможно точно описать разрыв плотности тока на границе ленты (см. [2]).

Рис. 5. Схема расположения магнитов в линейке относительно токовых линий в ленте. Каждая токовая линия соответствует току 3 А. Стрелка указывает направление движения линейки. Различной штриховкой обозначены магниты противоположной полярности. 


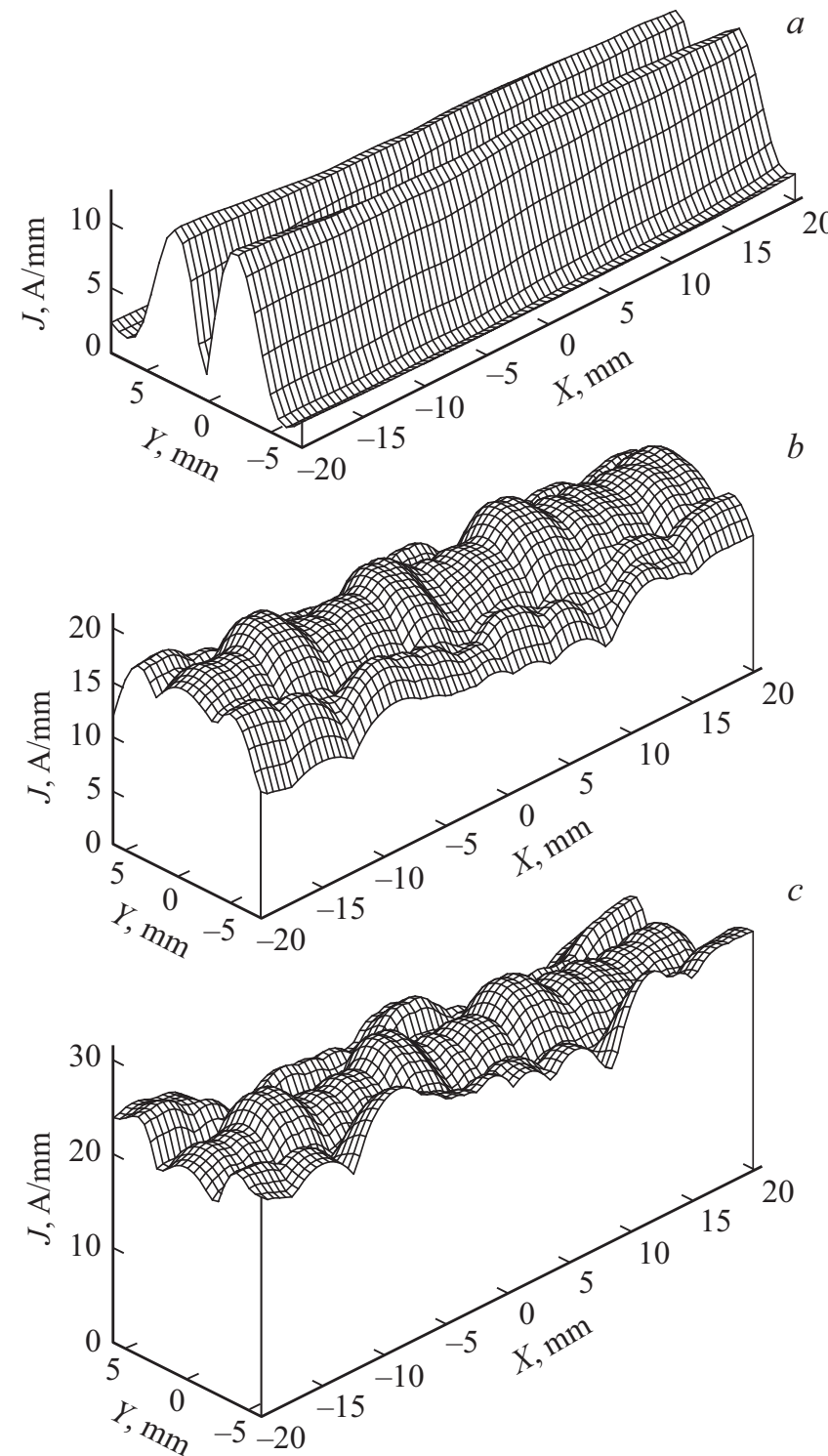

Pис. 6. Поверхностная плотность остаточного тока в ленте; $a$ - пространственное распределение при однородном намагничивании без корректировки границ, $b-$ пространственное распределение при неоднородном намагничивании без корректировки границ, $c$ - пространственное распределение при неоднородном намагничивании с корректировкой границ.

Третий недостаток стандартной методики связан с сильной зависимостью плотности критического тока $j_{c}(B)$ от магнитного поля $B$ при малых (до $\sim 0.5 \mathrm{~T}$ ) значениях этого поля. В двухэкспоненциальном приближении [8] для ҮВСО-ленты характерная зависимость плотности поверхностных токов имеет вид (8). Остаточная индукция намагниченной нами пленки в ее середине достигает величины $0.02 \mathrm{~T}$, что сопоставимо с величиной $\beta_{1}$, т.е. различные точки ленты находятся в существенно различных полях, а для целей диагностики однородности токовых характеристик необходимы одинаковые условия.
Первый и третий из перечисленных недостатков могут быть устранены намагничиванием ленты пространственно неоднородным полем. Для выбора оптимальной конфигурации ВМП в рамках двухэкспоненциального приближения (8) нами проведен предварительный модельный расчет распределения плотности токов (задача $(3)-(14))$ при однородном и неоднородном намагничивании. Из результатов предварительных расчетов выбрана процедура наведения остаточных токов при неоднородном намагничивании полем магнитной линейки, аналогичным представленному в выражении (13) (рис. 5). На рис.5 также представлены линии токов,
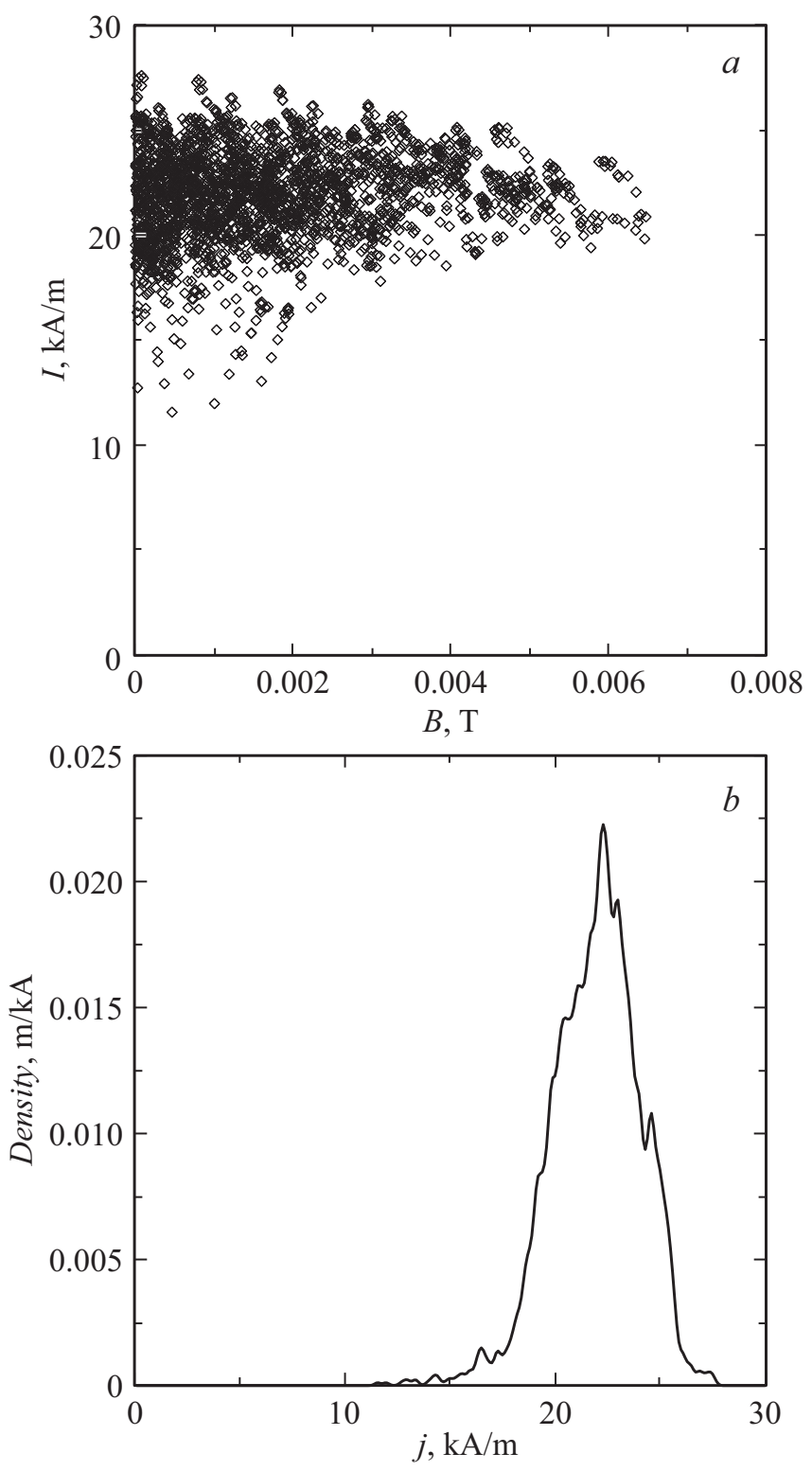

Рис. 7. Зависимость плотности критического тока в каждой точке расчетной сетки от значения нормальной компоненты магнитного поля в соответствующей точке $(a)$ и нормированная на единицу плотность распределения фрагментов ленты по величине плотности тока $(b)$. 
полученные из экспериментальных данных посредством решения обратной задачи Био-Савара (9).

Сопоставление результатов действия однородного и неоднородного ВМП показало большую эффективность последнего. При остаточной намагниченности ленты линейкой максимальное значение плотности тока в ленте превосходит аналогичную величину при однородном воздействии в $\sim 1.7$ раза. При этом максимальное значение наведенных токов наблюдается в области стыков соседних магнитов (там, где магнитное поле равно нулю), а токи направлены в поперечном направлении. Экспериментальное воспроизведение неоднородной намагниченности и последующее восстановление токов остаточной намагниченности полностью подтвердили результаты модельного расчета. На рис. 5 отчетливо наблюдаются ромбовидные токовые домены, токовые линии в которых параллельны друг другу и пересекают ленту в поперечном направлении. Проведение серии из нескольких намагничиваний линейкой, положение которой всякий раз смещается на небольшой шаг в продольном направлении (и последующее восстановление токов в каждом элементе серии), позволит определить максимально возможную плотность тока каждой точки ленты, причем в нулевом остаточном магнитном поле. Представленные ниже результаты получены для величины шага смещения в $1 \mathrm{~mm}$. Всего было проведено 21 измерение с различными продольными смещениями. Применение линейки именно таким способом позволяет в значительной степени устранить первый и третий недостатки традиционной методики.

Второй недостаток мы устранили, изменив численный алгоритм восстановления плотности токов по магнитному полю. Пусть расположение изучаемой ленты таково, что координаты ее точек в направлении $L_{Y} / 2<Y<L_{Y} / 2$ (координата поверхности ленты $Z=0$, а соответствующая высота расположения датчика Холла $\left.Z=h \ll L_{Y}\right)$. Плотность наведенных токов также отлична от нуля только в этом интервале. Заметим, что если пара $\mathbf{B}(X, Y, h)$ и $\mathbf{j}(X, Y, 0)$ удовлетворяет уравнению Био-Савара (1), то пара

$$
\begin{aligned}
\tilde{B}(X, Y, h)=B(X, Y, h) & -B\left(X,-L_{Y}-Y, h\right) \\
& -B\left(X, L_{Y}-Y, h\right)
\end{aligned}
$$

и

$$
\begin{aligned}
\tilde{j}(X, Y, 0)=j(X, Y, 0) & +j\left(X,-L_{Y}-Y, 0\right) \\
& +j\left(X, L_{Y}-Y, 0\right)
\end{aligned}
$$

также удовлетворяет этому уравнению. Определенная таким образом функция $\tilde{j}(X, Y, 0)$ отлична от нуля на интервале $3 L_{Y} / 2<Y<3 L_{Y} / 2$. На интервале $L_{Y} / 2<Y<L_{Y} / 2$, однако на границах ленты $Y=-L_{Y} / 2$ и $Y=L_{Y} / 2$ функция $\tilde{j}(X, Y, 0)$ m не испытывает разрыва, в отличие от исходного распределения токов $j(X, Y, 0)$. Вследствие большей гладкости функции $\tilde{j}(X, Y, 0)$ регуляризация при ее определении из $\tilde{B}(X, Y, h)$ даст меньшие искажения вблизи реальных границ ленты.
На рис. 6 представлена поверхностная плотность тока ленты при различных типах намагничивания. Coпоставление рис. $6, a, c, b$ и $c$ показывает, что методика неоднородного намагничивания позволяет получить заметно более высокую плотность тока за счет выбора областей с малым значением магнитного поля. Сопоставление рис. $6, b$ и $c$ показывает, что модификация методики восстановления позволяет подавить граничные искажения плотности тока, вследствие чего плотность тока в граничных областях заметно возрастает, а в центральной не изменяется. Неоднородность амплитуды наведенных токов на рис. 6, $c$ все же имеет место, однако уровень неоднородности принципиально более низкий, чем при традиционной методике на рис. 6, a. Рис. 7 иллюстрирует распределение плотности тока от величины магнитной индукции для каждого фрагмента ленты размером $0.5 \times 0.5 \mathrm{~mm}$ на основе данных рис. $6, c$. Также на рис. 7 представлена величина density $(j)-$ нормированная на единицу плотности распределения фрагментов по величине плотности тока. Рис. 7 показывает, что основная цель методики достигнута практически во всех представленных точках магнитное поле мало. На рис. 7 видно, что основная масса точек имеет плотность тока $J \sim 22 \mathrm{kA}$ и локализована в области поля $B<0.005 \mathrm{~T}$, что заметно меньше параметра $\beta_{1}=0.08 \mathrm{~T}$, вследствие чего основное „облако“ точек ожидаемо вытянуто параллельно оси $B$. К отрицательным чертам новой методики следует отнести больший объем экспериментальных измерений и необходимость задания координат краев ленты, однако существенное повышение точности результатов оправдывает эти недостатки.

Для дальнейшего повышения точности определения плотности критического тока перспективной выглядит замена остаточной намагниченности после удаления линейки, исследованием намагниченности ленты в фоновом поле линейки без удаления последней.

\section{Заключение}

Таким образом, в работе впервые представлены и проанализированы две различные взаимодополняющие методики диагностики дефектов ВТСП-лент, объединяющим элементом которых является использование градиентного магнитного поля, наводящего сильно неоднородные сверхпроводящие токи в изучаемых образцах. В первой из них исследованы возможности применения левитационной методики для диагностики трещин ВТСП-лентах и дефектов стопок из ВТСП-лент. В рамках модели, адекватно описывающей взаимодействие системы токов ВТСП-ленты с внешним неоднородным магнитным полем, определена левитационная сила взаимодействия стопки ВТСП-лент с линейкой постоянных магнитов противоположной полярности. Получены зависимости силы взаимодействия от расстояния между стопкой и линейкой магнитов при различном числе лент 
в стопке $M=1,2, \ldots, 50$. Показано, что при нулевом расстоянии между линейкой и стопкой лент сила их отталкивания пропорциональна количеству лент, если это количество не превышает $\sim 15$ лент. Если расстояние между линейкой и стопкой лент равно $1 / 3$ ширины ленты, сила их отталкивания пропорциональна количеству лент, если это количество не превышает четырех лент. Показано, что поперечная трещина даже в одной ленте стопки может заметно снизить силу левитации. Таким образом, данный эффект может быть использован для определения места локализации трещины. На основании полученных расчетных данных можно заключить, что такая диагностика будет эффективна, если число лент в стопке не превышает 5-10, а расстояние между стопкой лент и магнитной линейкой не превышает половины ширины ленты. Кроме диагностики трещин в стопке лент данная методика может применяться при определении местоположения трещины в обмотке сверхпроводящего соленоида, если количество слоев ВТСП-ленты в нем не превышает 10.

Вторая методика направлена на исследование слабонеоднородных ВТСП-лент. Данная методика позволяет проводить измерения плотности критического тока в любой точке ВТСП-ленты при фактически нулевом значении нормальной составляющей магнитного поля, что не реализуется в традиционных магнитометрических подходах. Модификация алгоритмической компоненты данной методики позволило заметно повысить точность определения критического тока по сравнению с существующими подходами.

Настоящая работа выполнена за счет гранта Российского научного фонда (проект № 17-19-01527).

\section{Список литературы}

[1] Токонесущие ленты второго поколения на основе высокотемпературных сверхпроводников / Под. ред. А. Гояла. М.: Изд-во ЛКИ, 2009. 432 с.

[2] Jooss Ch., Albrecht J., Kuhn H., Leonhardt S., Kronmuller H. // Rep. Prog. Phys. 2002. Vol. 65. P.651-788.

[3] Bean C.P. // Phys. Rev. Lett. 1962. Vol. 8. N 6. P. 250-253.

[4] Bean C.P. // Rev. Mod. Phys. 1964. Vol. 36. P. 31-39.

[5] Schuster Th., Kuhn H., Brandt E.H. et al. // Phys. Rev. B. 1995. Vol. 52. N 14. P. 10375-10389.

[6] Rudnev I.A., Podlivaev A.I. // IEEE Transactions on Applied Superconductivity. 2016. Vol. 26. N 4. 8200104-4.

[7] Podlivaev A., Rudnev I. // Supercond. Sci. Technol. 2017. Vol. 30. P. 035021-9.

[8] Подливаев А.И., Руднев И.А., Шабанова Н.П. // Краткие сообщения по физике ФИАН. 2014. Т. 41. Вып. 12. С. 1318.

[9] Vestgarden J.I., Mikheenko P., Galperin Y.M., Johansen T.H. // New J. Physics. 2013. Vol. 15. P. 093001-26.

[10] Подливаев А.И., Покровский С.В., Анищенко И.В., Руднев И.А. // Письма в ЖТФ. 2017. Т. 43. Вып. 24. С. 96-103.

[11] Sotelo G.G., Dias D.H.J.N., De Oliveira R.A.H. et al. // J. Physics: Conf. Series. 2014. Vol. 507. N 3. P. 032017-5.
[12] Deng Z., Zhang $W$., Zheng $J$. et al. // IEEE Transactions on Appl. Supercond. 2017. Vol. 27. N 6. P. 3602008-8.

[13] Werfel F.N., Floegel-Delor U., Rothfeld R. et al. // Supercond. Sci. Technol. 2012. Vol. 25. N 1. P. 014007-5.

[14] Pokrovskiy S.V., Ermolaev Y.S., Rudnev I.A. // Progress in Superconductivity and Cryogenics (PSAC). 2015. Vol. 17. N 1. P. 14-16.

[15] Rudnev I., Abin D., Osipov M., Pokrovskiy S., Ermolaev Y., Mineev N. // Phys. Procedia. 2015. Vol. 65. P. 141-144.

[16] Abin D., Osipov M., Pokrovskii S., Rudnev I. // IEEE Trans. Appl. Supercond. 2016. Vol. 26. N 3. P. 8800504-4.

[17] Pokrovskii S., Abin D., Osipov M., Rudnev I. // IEEE Trans. Appl. Supercond. 2016. Vol. 26. N 3. P. 8201304-3.

[18] Osipov M., Abin D., Pokrovskii S., Rudnev I. // IEEE Trans. Appl. Supercond. 2017. Vol. 27. N 4. P. 7815302-4.

[19] Neilinger P., Oelsner G., Grajcar М., Иванов Б.И., Новиков И.Л., Ильичев Е.В. // Письма в ЖТФ. 2015. Т. 41. Вып. 7. С. 16-21.

[20] Feldman D.M. // Phys. Rev. B. 2004. Vol. 69. N 14. P. 144515. 